\title{
User Interface Design for a Navigation and Communication System in the Automotive World
}

\author{
Olaf Preißner \\ Manager Design, \\ Harman/Becker Automotive Systems GmbH \\ Raiffeisenstr. 34 \\ 70794 Filderstadt, Germany \\ Tel: +49 / (0) $711 / 90770-551$ \\ opreissner@harmanbecker.com
}

\begin{abstract}
What makes a "smart graphic" and what distinguishes it from other design or graphic variants? Many existing designs for navigation and communication systems in high-end cars are not so attractive, don't feel the user emotional and are not easy to use. In contrast, smart graphics have the advantage that it entice the user to interact with the display, the systems or the application and appealing to his aesthetic expectations while maintaining high quality appearance. It is totally user-friendly and uncomplicated thus avoiding misapplication. Smart graphic uses real time execution of all functions ensuring simple and fast interaction. A limiting factor of performance is the capacity of the computer systems so a graphic has to adapt to the characteristics of the medium used. In the end, it also must satisfy high design expectations and technical features. The aim of this paper is to investigate the potential of 'smart graphics' in the design of user interfaces for automotive navigation and communication systems. A comparison will be made with other design and graphic variants to fully grasp the additional value of 'smart graphics'. Our experience has taught us that a smart graphic integrates all of these aspects while optimally combining aesthetic qualities, user specific conditions and the environment it is used in (e.g. in a vehicle). These insights are of great importance to arrive at interface designs that fully adhere to technical, aesthetic, emotional and usability requirements.
\end{abstract}

\section{The Driver Information and Communication System}

Driver information and communication systems must strictly comply with these characteristics to effectively display accessible information in the vehicle. Harman/Becker has developed such a system together with an interdisciplinary team of developers, designers and ergonomic scientists.

It is taken for granted that almost every car today has a radio, CD player and navigation system. This trend is steadily continuing. Increasingly, services which are used in the office or at home, such as Internet or email, are being transferred and modified specifically to fit into the unique environment of a car. The aim is to offer a logical and structured integration of the desired functions through the help of display technology. Complex driver information and communication systems combine 


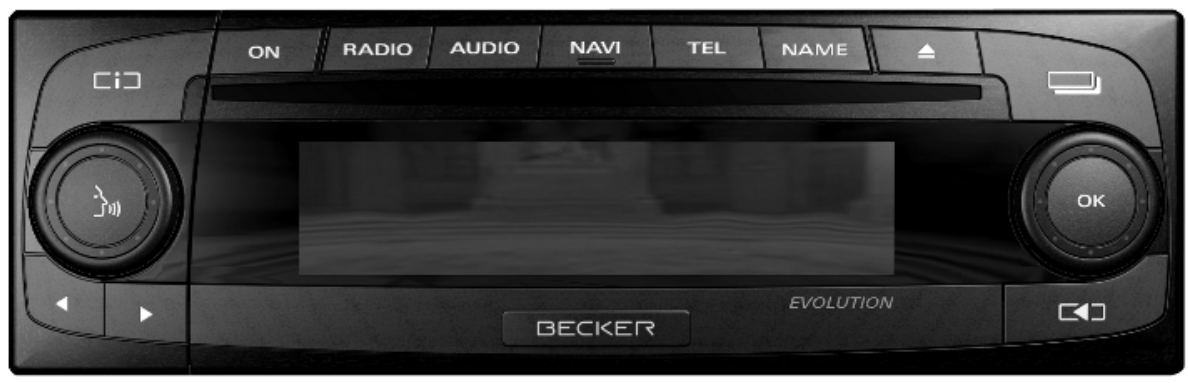

Fig. 1. New driver information system with speech recognition

individual components such as radio, $\mathrm{CD}$, navigation, telephone into a single integrated unit, enabling the user to interact using logical interface with high quality displays. As a general principle, all functions of the various components in an automobile are handled by a small number of controls require the driver's close attention to operate and are often difficult to see. This restricts the overall interaction with the system.

In order to make driver information systems for vehicles safe and comfortable the technology of speech recognition is a very important factor (Fig. 1). Using voice commands a driver doesn't have to look away from the road or take his hand off the steering wheel to access the navigation system or the car telephone. Additionally, by using speech for system in and output new options can be integrated such as listening to incoming emails while driving. Unfortunately, the technology in speech recognition systems hasn't been fully perfected and therefore, it can't be used alone in operating driver information systems. As mentioned above, complex situations are created when competing demands are placed on a driver of an automobile, these demands can only be resolved with an optimally configured smart graphic. The graphic design "user interface" is therefore devised by integrating the following aspects:

\section{Demands of the Medium, the Surroundings, and the Graphic Realization}

Operating a vehicle in traffic always demands high concentration and quick reactions by the driver. This means crucial information, which should be taken in parallel to driving, must be quickly recognized, understood, and processed.

Even complex components and system applications like navigation, air conditioning or the Internet in the future need to be operated using simple input elements. The frequently deeply branched out and involved menu structures are necessary to access all functions of the system, poses a unique challenge.

While driving it is important to localise all instruments on the cockpit quickly and easily. The better the display is placed in the cockpit the easier it is for the driver to read the indicated information. The driver must find the desired information in split seconds. Because of the better resolution in the middle retina the information can be 
better identified then the information in the lateral field of view, when the display is placed centrally placed in the cockpit [1].

Unfortunately, only in exceptional cases is it possible to place ideally the display. The packaging in the car such as the climate channels in the cockpit, are limit the positioning of the display. Since the location cannot always meet optimal ergonomic demands, the information must also be absorbed from a limited viewing angle or from a considerable distance.

The "Ergonomic aspects of in-vehicle visual presentation of information" from the International Organisation for standardization recommends specific types and sizes of fonts to optimize the readability during driving [2]. Also extreme lighting situations, during the day or at night, can drastically reduce readability of the display. Varied lighting conditions greatly affect the display contrast resulting in poor readability of the display.

\section{Demands of the Driver}

These restrictions conflict with the main desires of the driver for ease of operation and elegant design. The user expects a graphic design that follows logical rules, integrates and reflects his environment, expectations and thought processes as much as possible, that also encourages him to interact. Operating must be made to be simple and intuitive; the driver shouldn't have to concentrate when he asks for information in a timely manner.

Since such a system is built mainly into upper class automobiles, the design of the user interfaces must complement the high aesthetic standard of the rest of the car's interior that the driver is accustomed to.

The top priority for developing a graphic concept is to follow the design guide lines that the customer is used to having. The design of the interface must convey this message, such as representing the company's philosophy, and fitting into the respective vehicle.

Also, different drivers have distinct patterns of habit and action. It is therefore already essential to differentiate the users' specifics in the conceptional phase and the design of the interface. As an example we can consider the group of older traffic participants. The more complex the information is designed and the more tasks are need to resolved at the same time, the more inhibited is the information processing by older people [3]. We can assume that older people have a general, old-age worsening visual acuity [4]. Examinations by B. Bell, E. Wolf and C.D. Bernholz [5] show, for example a decreasing of the depth perception between the age of 40 and 50 . Furthermore, J.G. Gilbert [6] was found out that there is a reduction in sensitivity to the color spectrum of about $24 \%$ between the ages $60-70$. The reading precisions from displays could be strong assuaged by designing the screens adequate. This kind of flexibility in operating the system can be achieved by a design that the user can tailor to his specific desires. 


\section{The Design of the System's Graphic Display}

The expectations of the driver as well as the demands of the system have to combine using a smart graphic in order to satisfy the above mentioned needs.

The exterior and interior design create the individual statement of a vehicle type. In the interior of the vehicle the active communication surfaces are an important part of this statement even though they only take up a relatively small part of the interior. It is therefore essential to adhere to the established guidelines and to unify them.

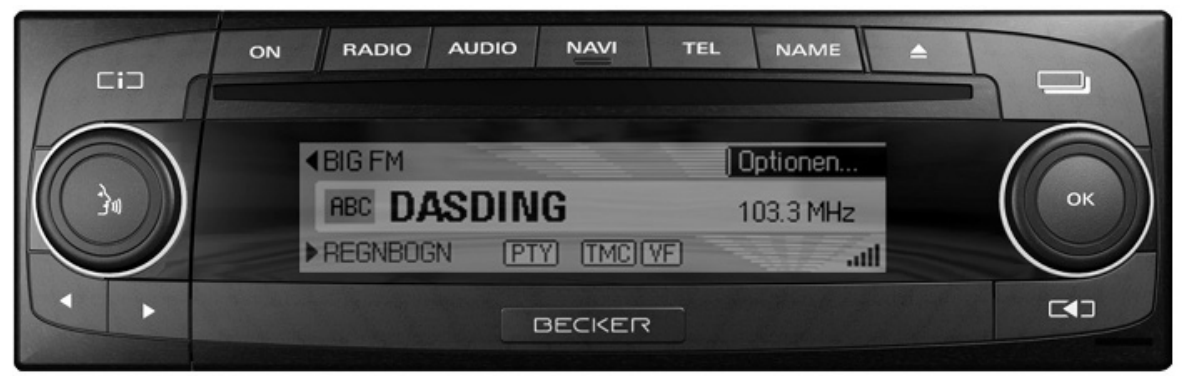

Fig. 2. One hardkey for each function permitts a flat menu hierarchy

In order to simplify the menu guide, flat menu hierarchies are being developed that demonstrate clearly separated menu points and distribution of the various functions. The contents of the individual categories is dependent on the different components in the vehicle and the needs of the user, such as the division of the main menu into radio, audio, navigation, telephone, information and address book (Fig. 2).

It is essential that the menu structure is consistent and well organized. Only in this way intuitive operation is possible and therefore an effortless orientation of the driver is guaranteed. The need for an easily understandable graphic is required to make up for limiting the main menu and greatly reducing structure logic.

To enable intuitive use, the designed form of the user elements is embody into the design of the graphic display of the user interface. This system uses a rotary input device with a push function. That means that the input elements are reflected in the design and give the driver the feeling that he initiates actions directly in the display. If a user element is turned to the right the cursor on the screen also moves to the right. Basically, the direction of movement of the user element and the screen can't conflict, instead they should correspond with each other.

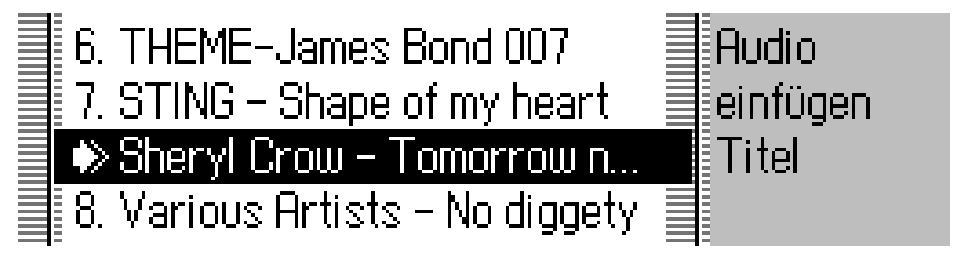

Fig. 3. Different cursor while storing a station 
Next to the formal design, another deciding factor is the color-coding of the different main menus. Using bright and different color choices create easy orientation even under unfavourable viewing conditions. The user knows immediately which menu he is viewing. This is the reason why the cursor, used to mark the actual position of the input element, is identified clearly by color and significant contrast with the background making it immediately visible no matter what the lighting conditions are. The cursor is simply the navigation tool, marking the current position on the screen and is the reference point for the next action of the user. The cursor can change into different forms that express different statements, informing the user of the next possible step (Fig. 3).

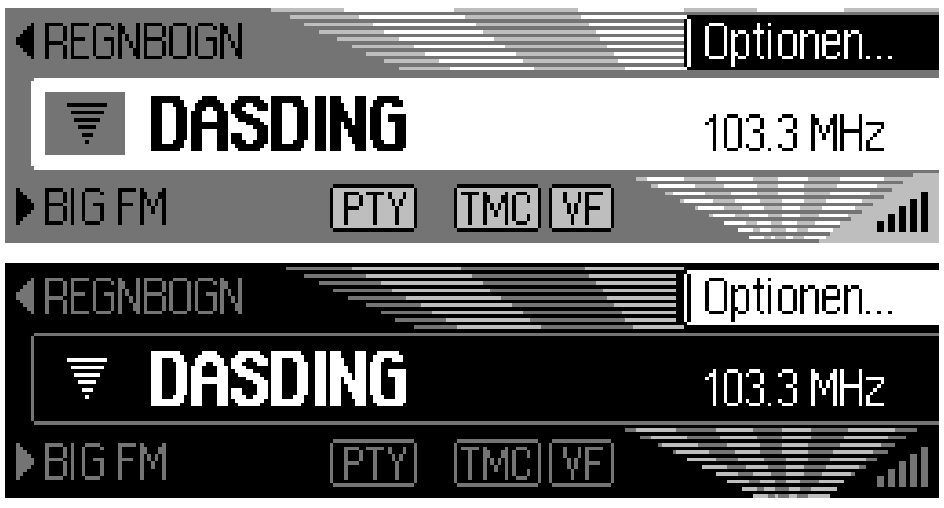

Fig. 4. Day and night design

The manipulation of light and shadow, such as use of contrast values, enable optimal readability even in unfavourable light conditions like the sun shining through the back window. One option in this area is to create a day and night design (Fig. 4). Depending on the time of day and variations in brightness an optimal graphic display is offered to the driver, which is adapted to the specific characteristics of the incoming light conditions.

During daylight a brighter screen is considered more comfortable to read. Since the eyes aren't strained by uneven lighting conditions, less fatigue is observed that with a dark screen during daylight. Uncomfortable light reflections are also avoided by using a bright screen background. At night darker colors are more appropriate so the driver isn't blinded. The contrasts can be reduced in the night screen design since a screen designed for daylight tends to be too bright under dark conditions.

Next to a rich contrast display, the typographic on the display play an important role. Due to the more difficult sensory environment encountered in a vehicle, the font used in the display has to adhere to stringent rules in order to achieve the primary goal of optimal readability. Important criteria for the suitable typographic are the font type, cut, size and running width. Fonts without serifs (Arial, Helvetia, Univers, Frutiger) are preferred over those having serifs (like Times and Bodoni). Regular or bold font cut is best suited, while cursive or condensed fonts should be avoided. The font size depends on a number of factors such as viewing distance, form, contour or contrast. Having chosen an optimal font type for form, contour and contrast a font size of at 
least $5 \mathrm{~mm}$ for capital letters is required when viewing from a $70 \mathrm{~cm}$ distance. Smaller font sizes are acceptable for non-critical messages or status indications, which do not need to be viewed while driving. Besides the criteria of readability the font should also reflect the corporate identity of the brand and accent the appearance.

A decisive factor is also the differentiation between an information display, which shows status information and that of active display elements that are triggered when initiating a certain action. Menu levels are set in theme-related backgrounds and therefore, the function lying behind it visualized. For totally new functions a new symbolic system must be developed that intuitively makes sense without having to refer to an operator's manual.

In order to be able to use the symbolic system successfully it has to be absolutely indisputable. Icons have to always be recognizable and readable and to the greatest degree adhere to the common standard or DIN norms. The meaning of the icons has to be universally recognized. Many symbols with an established meaning in Europe may be interpreted differently by other cultures. Example: In Europe the analog clock is the symbol for the dimension of "time", in Asia this is the symbol for "death".

In order to be able to understand the statically displayed information with minimal effort, small and much reduced animations are used that provides direction to the user, thus permitting easier navigation. An example for this application is the animation of the repeat function in the audio menu (Fig 5).

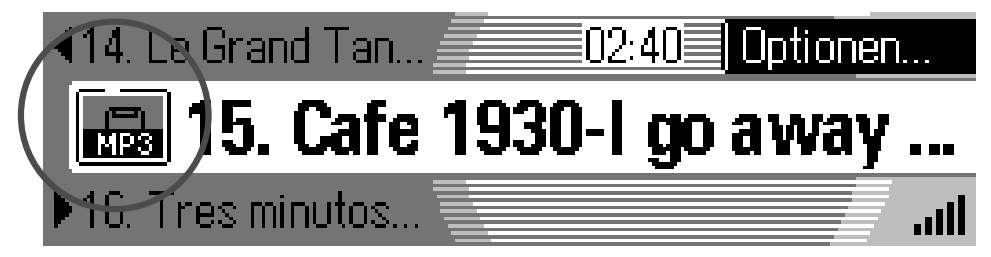

Fig. 5. Animated MP3 repeat function

The arrangement of the graphic elements in a linear fashion throughout all hierarchy levels is the basic requirement for all screens of the system. Even though the layout configuration is strictly fixed, the arrangement of information along with text fields must be flexible enough in order to conform to other language variants requiring a different number of symbols. Here, the system will look for the smallest common denominator in flexibility between the number of the symbols and retaining the page arrangement (Fig. 6)

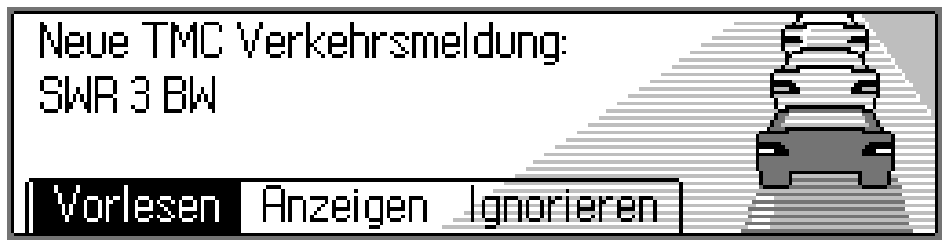

Fig. 6. Flexible text fields for different languages

A test with 13 people showed improved user interaction with the interface design discussed above. A formative usability test of the interaction principles and concept 
has been carried out. In the study 13 people, 8 male and 5 female, took part. The test persons were between 22 and 44 years old. Nine test persons hadn't had experience with navigation systems, the other 4 had used navigation systems before. The tasks comprised often used applications like radio, telephone, audio and navigation without any guidance or support. This new system compared with a similar driver information system with a smaller display, (Becker Online Pro) was the starting point for the benchmark. The analysis of the subjective evaluation shows a decided preference for the new system with the smart graphics. The key points were: positive overall impression, aesthetic design, attractive color, good readability from a distance, clearly arranged screen design, further there were easy to use, not complicated and generally user friendly.

The uniqueness of the above described graphic is that it not only considers the specific factors of the environment it is being used in but places great emphasis on aesthetic expectations and being user friendly. All these unique characteristics and distinctions make for a optimally sized graphic that is logical and to the point, but at the same time not boring or unimaginative - just a smart graphic.

\section{References}

1. Hollwich, F.: Augenheilkunde, Thieme, Stuttgart 1988

2. ISO TC22/SC13/WG8 "TICS M.M.I". Road vehicles - Traffic information and control systems - ergonomics aspects of in-vehicle visual presentation of information.

3. Welford, A.T.: Aging and human skills, Greenwood Press, Connecticut, 1975

4. Cohen,A.S.: Sensorik und ihre altersabhängige Variation. In U. Tränkle, 1994

5. Bell, B., Wolf, E. \& Bernholz, C.D.: Depth perception as a function of age, Aging and Human Development, (77-88), 1972

6. Gilbert,J.G.: Age changes in colour matching. Journal of Gerontology, 210-215, 1957

7. Apple Computer Inc, Human Interface Guidelines, The Apple Desktop Interface, AddisonWesley Publishing Company, Bonn Paris, 1987

8. Bühl Achim: Cyber Society, Mythos und Realität der Informationsgesellschaft, PapyRossa Verlags GmbH \& Co. KG, Köln, 1996

9. Brugger, Ralf: Professionelle Bildgestaltung in der 3D Computergrafik, Addison-Wesley, Bonn, 1995

10. Cyrus Dominik Khazaeli: Typo und Layout, Vom Zeilenfall zum Screendesign, Rowohlt Taschenbuch Verlag GmbH, 1995

11. Hannemann Jörg, Thüring Manfred: Designing User Interfaces for Hypermedia, Springer Verlag, Berlin Heidelberg, 1995

12. Helander M.: Handbook of Human-Computer Interaction, Elsevier Science Publishers, Amsterdam, 1988

13. Kieselbach Ralf: The drive to design, av-Ed., Stuttgart 1998

14. Schneiderman B.: Designing the User Interface, Strategies for Effective Human-Computer Interaction, Addison-Wesley, 1992

15. Stary Christian: Interaktive System; Software-Entwicklung und Software-Ergonomie, Vieweg Verlag, 1996

16. Stary, Joachim: Visualisieren, ein Studien- und Praxisbuch, Cornelsen Scriptor, Berlin, 1997

17. Human Factors Design Guidlines for Advanced Traveler Information Systems (ATIS) and Commercial Vehicle Operations (CVO), U.S. Department of Transportation, Federal Highway Administration, Report FHWA-RD-98-057, 1998 